\title{
IM AUSLAND GEBORENE UND AUSLÄNDISCHE BEVÖLKERUNG
}

Auf Grund unterschiedlich starker Migrationsströme in verschiedene Zielländer variiert der Anteil der Zuwanderer und der ausländischen Bevölkerung von einem Land zum anderen. Die genaue Definition dieser Anteile ist von entscheidender Bedeutung, um internationale Vergleiche anzustellen.

\section{Definition}

Staatsangehörigkeit und Geburtsland sind die beiden zur Definition der „zugewanderten“ Bevölkerung am häufigsten verwendeten Kriterien. Bei der im Ausland geborenen Bevölkerung handelt es sich um Menschen, die im Lauf ihres Lebens von ihrem Geburtsland in das Land ihres aktuellen Wohnsitzes umgesiedelt sind. Die ausländische Bevölkerung setzt sich demgegenüber aus den Personen zusammen, die noch die Staatsangehörigkeit ihres Herkunftslands besitzen. Dazu können auch Personen gehören, die im Aufnahmeland geboren sind.

\section{Vergleichbarkeit}

Die im Ländervergleich bestehenden Unterschiede im Hinblick auf den Umfang der im Ausland geborenen Bevölkerung und der ausländischen Bevölkerung hängen von den in den jeweiligen Ländern geltenden Regeln für den Erwerb der Staatsbürgerschaft $a b$. In einigen Ländern erwerben die dort geborenen Kinder automatisch die Staatsbürgerschaft des Geburtslands (ius soli, „Recht des Bodens“, also Geburtsrecht), in anderen Ländern hingegen ist die Staatsbürgerschaft der Eltern maßgeblich (ius sanguinis, „Recht des Bluts“, also Abstammungsprinzip). In einigen anderen Ländern behalten sie bis zur Volljährigkeit die Staatsbürgerschaft ihrer Eltern, können dann aber die des Aufnahmelands erhalten. Das Auseinanderklaffen der beiden Datenreihen erklärt sich z.T. daraus, wie leicht oder schwer Zuwanderern der Erwerb der Staatsbürgerschaft des

\section{Überblick}

Der Anteil der im Ausland geborenen Bevölkerung an der Gesamtbevölkerung ist in Luxemburg, Australien, der Schweiz, Israel, Neuseeland und Kanada besonders hoch, wo er zwischen $21 \%$ und $42 \%$ liegt. In einer Reihe anderer europäischer Länder (namentlich Belgien, Spanien, Irland, Deutschland, Estland, Österreich und Schweden) ist der Anteil höher als in den Vereinigten Staaten (13,1\%). Er ist im vergangenen Zehnjahreszeitraum in allen Ländern gestiegen, für die Daten verfügbar sind, mit Ausnahme der zwei jüngsten OECD-Mitgliedsländer Estland und Israel.

Der Anteil der im Ausland geborenen Bevölkerung an der Gesamtbevölkerung hat sich in diesem Zeitraum in Spanien, Irland und Norwegen ungefähr verdoppelt. Andere Länder wie Finnland, Südafrika und Chile weisen einen niedrigen Anteil im Ausland geborener Einwohner an der Gesamtbevölkerung auf, verzeichneten aber in den vergangenen Jahren einen spektakulären Anstieg. Die ausländische Bevölkerung wächst demgegenüber langsamer, weil die Zuwanderung neuer ausländischer Staatsangehöriger in der Regel durch Einbürgerungen aufgewogen wird. jeweiligen Aufenthaltslands gemacht wird. Die erforderliche Wohnsitzdauer für den Erwerb der Staatsbürgerschaft variiert z.B. zwischen nur drei Jahren in Kanada und bis zu zehn Jahren in einigen anderen Ländern.

In klassischen Einwanderungsländern, wie Australien, Kanada und Neuseeland, sowie in einigen europäischen Ländern, wie Belgien, Schweden und den Niederlanden, ist eine hohe Einbürgerungsrate $\mathrm{zu}$ beobachten. Im Allgemeinen ist der Prozentsatz der Zuwandererbevölkerung bei Anwendung des Kriteriums der Geburt im Ausland wesentlich höher als nach der Definition auf der Basis des Nationalitätskonzepts, weil viele im Ausland geborene Personen die Staatsangehörigkeit des Aufnahmelands annehmen und nicht mehr in den Ausländerstatistiken erscheinen. Das Geburtsland bleibt hingegen dasselbe, sofern es nicht zu Änderungen im Grenzverlauf kommt.

Die meisten Daten für diesen Indikator sind den Beiträgen der nationalen Korrespondenten entnommen, die Teil der OECDExpertengruppe zur Internationalen Migration sind.

In den Daten zur im Ausland geborenen Bevölkerung sind auch Personen erfasst, die im Ausland als Staatsangehörige des Landes ihres aktuellen Wohnsitzes geboren sind. In einigen Ländern kann diese Gruppe recht groß sein, namentlich in Frankreich und Portugal, die hohe Zuzüge von Repatriierten aus den ehemaligen Kolonien verzeichneten.

\section{Quelle}

- OECD (2012), International Migration Outlook, OECD Publishing. Weitere Informationen

\section{Analysen}

- OECD (2011), Tackling the Policy Challenges of Migration, Regulation, Integration, Development, Development Centre Studies, OECD Publishing.

- OECD (2008), A Profile of Immigrant Populations in the 21st Century: Data from OECD Countries, OECD Publishing.

- Widmaier, S. und J-C. Dumont (2011), "Are Recent Immigrants Different? A New Profile of Immigrants in the OECD based on DIOC 2005/06", OECD Social, Employment and Migration Working Papers, No. 126.

\section{Statistiken}

- OECD (2012), Connecting with Emigrants, A Global Profile of Diasporas, OECD Publishing.

- OECD (2012), Integration von Zuwanderern: OECD-Indikatoren 2012, OECD Publishing.

\section{Zur Methodik}

- Lemaître, G. und C. Thoreau, (2006), Estimating the foreign-born population on a current basis, OECD, Paris.

\section{Online-Datenbanken}

- OECD International Migration Statistics.

Websites

- Database on Immigrants in OECD Countries (DIOC), www.oecd.org/els/migration/dioc. 
Im Ausland geborene und ausländische Bevölkerung

\begin{tabular}{|c|c|c|c|c|c|c|c|c|c|}
\hline & \multicolumn{8}{|c|}{ In Prozent der Gesamtbevölkerung } & \multirow{3}{*}{\begin{tabular}{|c|}
$\begin{array}{c}\text { In Prozent aller im } \\
\text { Ausland Geborenen }\end{array}$ \\
$\begin{array}{l}\text { Im Ausland geborene } \\
\text { Staatsangehörige }\end{array}$ \\
$\begin{array}{l}2010 \text { oder letztes } \\
\text { verfügbares Jahr }\end{array}$ \\
\end{tabular}} \\
\hline & \multicolumn{4}{|c|}{ Im Ausland geborene Bevölkerung } & \multicolumn{4}{|c|}{ Ausländische Bevölkerung } & \\
\hline & 1995 & 2000 & 2005 & 2010 & 1995 & 2000 & 2005 & 2010 & \\
\hline Australien & 23.0 & 23.0 & 24.2 & 26.8 &.. & . & .. &.. & .. \\
\hline Belgien & 9.7 & 10.3 & 12.1 & 14.4 & 9.0 & 8.4 & 8.6 & 9.2 & 44.6 \\
\hline Chile & 0.9 & 1.2 & 1.4 & 1.9 & .. & & & .. & .. \\
\hline Dänemark & 4.8 & 5.8 & 6.5 & 9.6 & 4.2 & 4.8 & 5.0 & 5.4 & 46.7 \\
\hline Deutschland & 11.5 & 12.5 & 12.6 & 16.4 & 8.8 & 8.9 & 8.8 & 9.0 & 55.1 \\
\hline Estland & .. & 18.4 & 17.5 & 16.6 & .. & .. & .. & 17.6 & 35.7 \\
\hline Finnland & 2.1 & 2.6 & 3.4 & 3.7 & 1.3 & 1.8 & 2.2 & 2.1 & 47.2 \\
\hline Frankreich & .. & 10.1 & 11.0 & 12.6 & .. & .. & .. & 5.9 & 53.4 \\
\hline Griechenland & .. & .. & .. & 8.7 & .. & 2.9 & 5.2 & 7.0 & 22.1 \\
\hline Irland & .. & 8.7 & 12.6 & 15.5 & 2.7 & 3.3 & 6.3 & 11.2 & 29.9 \\
\hline Island & .. & .. & .. & 7.9 & .. & .. & .. & 3.7 & 54.7 \\
\hline Israel & .. & 32.2 & 29.1 & 31.2 & .. & .. & .. & .. & .. \\
\hline Italien & .. & .. & .. & 8.8 & 1.7 & 2.4 & 4.6 & 6.5 & 26.9 \\
\hline Japan & .. & .. & .. &.. & 1.1 & 1.3 & 1.6 & 1.7 & .. \\
\hline Kanada & 17.2 & 18.1 & 19.5 & 21.3 & .. & .. & .. & .. & .. \\
\hline Korea & & .. & .. & .. & 0.2 & 0.4 & 1.1 & 2.0 & .. \\
\hline Luxemburg & 30.9 & 33.2 & 35.0 & 42.4 & 33.4 & 37.3 & 39.6 & 41.8 & 13.2 \\
\hline Mexiko & 0.5 & 0.5 & 0.6 & 0.9 & .. & .. & .. & .. & .. \\
\hline Neuseeland & .. & 17.2 & 20.3 & 23.2 & .. & .. & .. & .. & .. \\
\hline Niederlande & 9.1 & 10.1 & 10.6 & 11.2 & 4.7 & 4.2 & 4.2 & 3.6 & 70.5 \\
\hline Norwegen & 5.5 & 6.8 & 8.2 & 10.0 & 3.8 & 4.0 & 4.8 & 5.6 & 49.0 \\
\hline Österreich & .. & 10.4 & 14.5 & 16.7 & 8.5 & 8.8 & 9.7 & 10.4 & 41.9 \\
\hline Polen & .. & .. & .. & 0.9 & .. & .. & .. & 0.1 & 87.1 \\
\hline Portugal & 5.2 & 5.1 & 6.3 & 7.3 & 1.7 & 2.1 & 4.1 & 3.5 & 53.9 \\
\hline Schweden & 10.6 & 11.3 & 12.5 & 16.8 & 6.0 & 5.4 & 5.3 & 5.6 & 67.7 \\
\hline Schweiz & 21.4 & 21.9 & 23.8 & 27.8 & 18.9 & 19.3 & 20.3 & 21.6 & 32.1 \\
\hline Slowak. Rep. & .. & .. & 4.6 & 0.7 & 0.4 & 0.5 & 0.5 & 0.2 & 74.4 \\
\hline Slowenien & .. & .. & .. & 9.1 & .. & .. & .. & 1.3 & 85.5 \\
\hline Spanien & .. & 4.9 & 11.1 & 14.9 & .. & .. & .. & 12.3 & 18.3 \\
\hline Tschech. Rep. & .. & 1.2 & 1.5 & 2.9 & .. & .. & .. & 1.1 & 63.1 \\
\hline Türkei & .. & 1.9 & .. & 3.8 & .. & .. & .. & .. & .. \\
\hline Ungarn & 2.7 & 2.9 & 3.3 & 1.9 & 1.4 & 1.1 & 1.5 & 0.6 & 69.6 \\
\hline Ver. Königreich & 6.9 & 7.9 & 9.4 & 12.9 & 3.4 & 4.0 & 5.2 & 7.4 & 41.9 \\
\hline Ver. Staaten & 10.1 & 11.5 & 13.3 & 13.1 & 6.1 & 6.5 & 7.5 & 7.1 & 54.0 \\
\hline EU27 & .. & .. & .. & .. & .. & .. & .. & .. & .. \\
\hline OECD & .. & .. & .. & .. & .. & .. & .. & .. & .. \\
\hline Brasilien & 0.4 & 0.4 & 0.4 & 0.4 &.. & .. & .. &.. & .. \\
\hline China & 0.0 & 0.0 & 0.0 & 0.1 & .. & .. & .. & .. & .. \\
\hline Indien & 0.7 & 0.6 & 0.5 & 0.4 & .. & .. & .. & .. & .. \\
\hline Indonesien & 0.1 & 0.1 & 0.1 & 0.1 & .. & .. & .. & .. & .. \\
\hline Russ. Föderation & 7.9 & 8.1 & 8.4 & 8.7 & .. & 8.2 & .. & .. & .. \\
\hline Südafrika & 2.7 & 2.3 & 2.6 & 3.7 & .. & 1.0 & .. & .. & 57.1 \\
\hline
\end{tabular}

\section{Im Ausland geborene Bevölkerung}

In Prozent der Gesamtbevölkerung

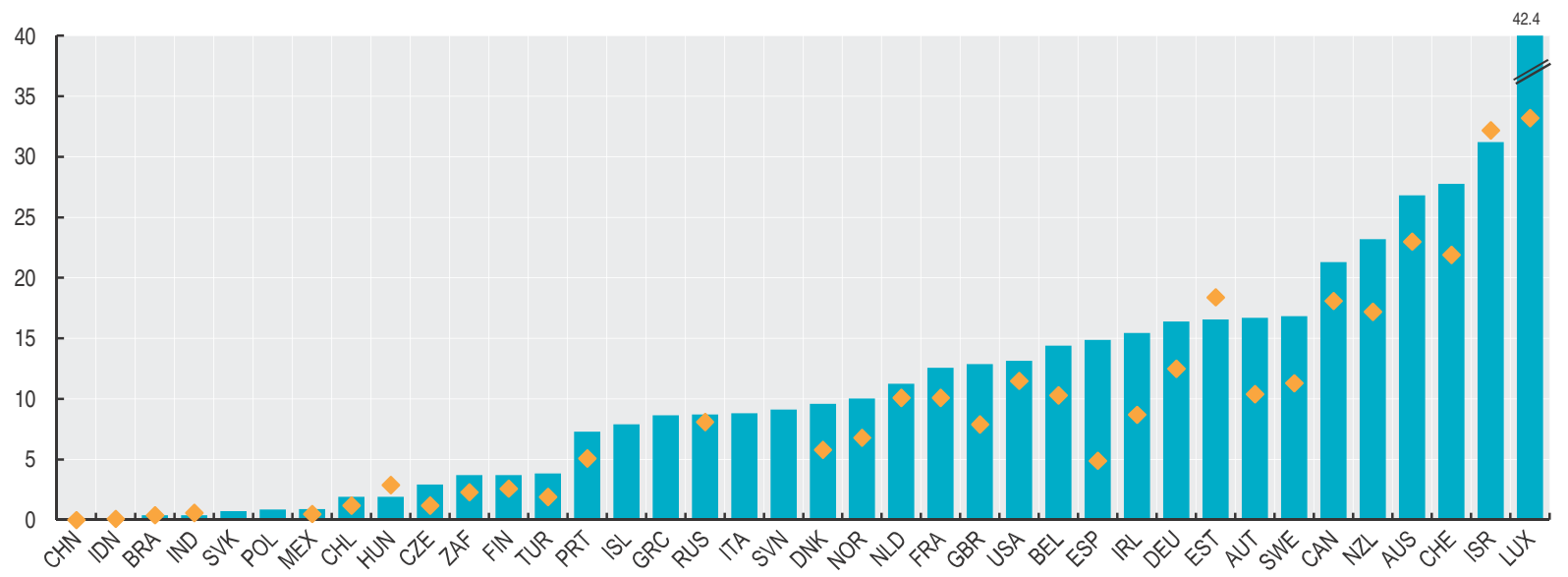




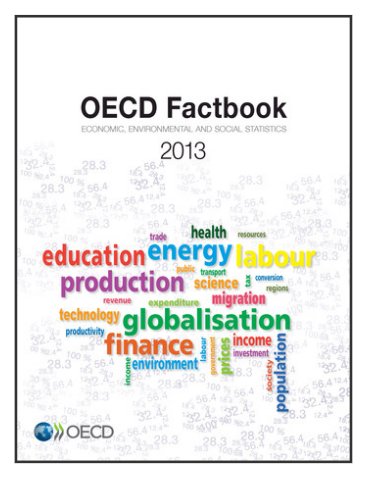

\section{From: \\ OECD Factbook 2013 \\ Economic, Environmental and Social Statistics}

Access the complete publication at:

https://doi.org/10.1787/factbook-2013-en

\section{Please cite this chapter as:}

OECD (2013), "Im Ausland geborene und ausländische Bevölkerung", in OECD Factbook 2013: Economic, Environmental and Social Statistics, OECD Publishing, Paris.

DOI: https://doi.org/10.1787/factbook-2013-6-de

Das vorliegende Dokument wird unter der Verantwortung des Generalsekretärs der OECD veröffentlicht. Die darin zum Ausdruck gebrachten Meinungen und Argumente spiegeln nicht zwangsläufig die offizielle Einstellung der OECDMitgliedstaaten wider.

This document and any map included herein are without prejudice to the status of or sovereignty over any territory, to the delimitation of international frontiers and boundaries and to the name of any territory, city or area.

You can copy, download or print OECD content for your own use, and you can include excerpts from OECD publications, databases and multimedia products in your own documents, presentations, blogs, websites and teaching materials, provided that suitable acknowledgment of OECD as source and copyright owner is given. All requests for public or commercial use and translation rights should be submitted to rights@oecd.org. Requests for permission to photocopy portions of this material for public or commercial use shall be addressed directly to the Copyright Clearance Center (CCC) at info@copyright.com or the Centre français d'exploitation du droit de copie (CFC) at contact@cfcopies.com. 